\title{
Wireless Thermocouple - Consequently from Input to Output
}

\author{
Binhack, Michael \\ senTec Elektronik $\mathrm{GmbH}$ \\ Werner-von-Siemens-Str. 6, D-98693 IImenau
}

\begin{abstract}
Wireless thermocouple for temperature measurement is well known since many years. But all available systems only mean a wireless transmission system with the ability to use thermocouples as input device. For output normally common digital and analogue interfaces like RS232, RS485, USB, CAN, ect. as well as $0 \ldots 10 \mathrm{~V}, 4 . .20 \mathrm{~mA}$ ect. are used. Up to now there exist no wireless solutions with thermo voltage output.

In different industrial and automotive applications many systems do not allow to replace the input interface. There is the strong demand of using thermo voltage as the only input signal. Especially for rotating or moving parts it is a great advantage to use wireless transmissions. Therefore a wireless system with the same behavior like a normal thermocouple has to be developed to fulfill the demand.

This paper shows the advantage of a real wire-less thermocouple with a generated thermo voltage as output signal. Especially the problems concerning the generation of a high precision voltage in the range of several $\mu \mathrm{V}$ will be discussed. Also the dynamical handling of the temperature behavior of different locations of the system parts including the different cold-junction compensations on both sides will be indicated. Finally the measurements and results of the test system under harsh environmental conditions in automotive tests will be presented.
\end{abstract}

\section{Introduction}

Especially in the automotive industry lots of temperature measurements were done with standard thermocouples (mostly type $\mathrm{K}$ or $\mathrm{J}$ ) since years. Therefore there exist an enormous distribution of measurement equipment for the usage of thermocouples. But also in the automotive industry the demand for wireless measurement and transmission systems to detect the temperature values out of rotating parts is rising strongly.

Therefore the first telemetering systems or wireless measurement systems were developed. Unfortunately with those wireless systems most of the common and well known measurement equipment cannot be used anymore because the output signal of the wireless system is not the same as the input signal of the common equipment. A solution which integrates the benefit of modern temperature measuring with wireless transmission and the usage of the well known equipment has to be developed. This will give maximum benefit for the user who still can use his common equipment.

\section{Measurements with Thermocouples}

Figure 1 shows a typical thermocouple (here type $\mathrm{K}$ ) which consists of a junction of two different metals (here Chromel and Alumel) which produces a voltage related to the temperature difference between the hot and the cold junctions [1].

The generated voltage can be measured with cables or connections out of a third metal (here copper) 


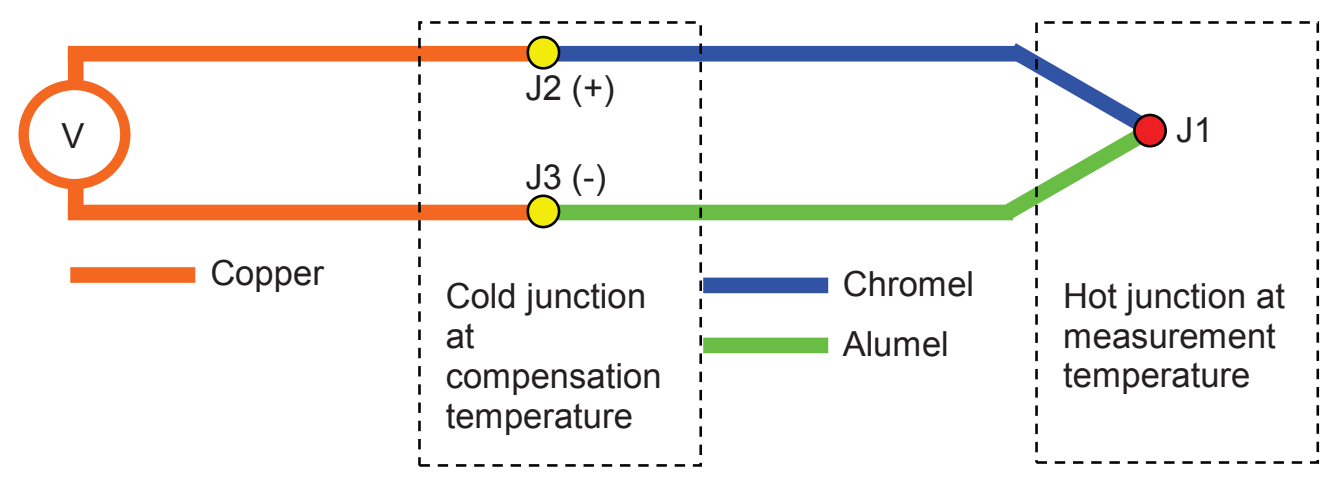

Figure 1: Thermocouple (Type K)

It is well known that the compensation temperature has to be measured too to determine the temperature at the hot junction. This is exactly what is done in nearly all common thermocouple measurement equipment.

\section{Wireless Thermocouple}

The wireless thermocouple is a system which uses a standard thermocouple (e.g. type K) as input, transmits the values wireless to a receiver, generates a correct thermo voltage as output to be connected to available common measurement equipment. Figure 2 shows this schematically.
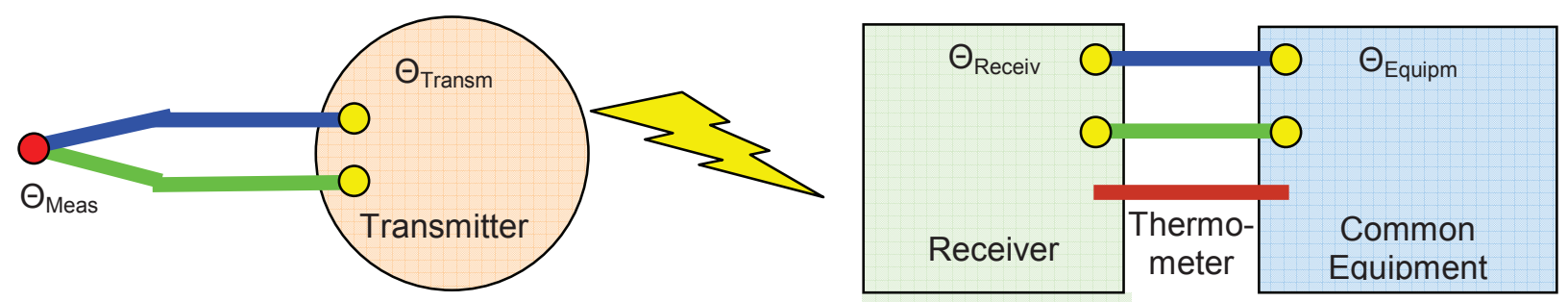

Figure 2: wireless thermocouple principle operation

The first part of the system works as a common measurement equipment. The voltage generated by the temperature difference between $\Theta_{\text {Meas }}$ and $\Theta_{\text {Transm }}$ will be detected as well as the temperature of the transmitter junctions. The measured values will be converted to digital values and transmitted by standard personal area network modules based on standard IEEE802.15.4 to the receiver [2]. This is very comfortable because a very robust and well proved radio link is used.

At the receiver side the temperature of the common equipment will be measured as well as the temperature of the junctions inside the receiver. A voltage of $\Theta_{\text {Meas }}$ related to $\Theta_{\text {Equipm }}$ will be generated out of the received values. Additionally the thermo voltage of the difference between $\Theta_{\text {Receiv }}$ and $\Theta_{\text {Equipm }}$ has to be taken into account to get proper values.

\section{Voltage Generation}

According to the fact that thermo voltage is in the region of several $\mathrm{mV}$ and varies $\sim 40 \mu \mathrm{V}$ per Kelvin (for thermocouples type $\mathrm{K}$ ) it is absolutely necessary to generate the voltage with minimum noise and minimum offset. Therefore only devices of high precision and low tolerances have to be used. A 
extremely stable output signal has to be generated because it is unknown which input impedance the connected devices will have.

Of course the connected common equipment will do a cold junction compensation as well because it is made for the usage of thermocouples. This has to be taken into account for voltage generation. It is very important to know the temperature of the junction inside the connected common equipment. Therefore the usage and the mounting place of the external thermometer as near as possible to the junction leads to a big influence of the system behavior. It is recommended to use wires out of one metal. If compensating cables are used it is very important to measure the temperature difference of its junctions to take the additionally generated thermo voltage into account.

The generated voltage is related to the following temperatures and their thermo voltages of the used couple:

$$
\begin{aligned}
& \Theta_{\text {Meas }} \\
& \Theta_{\text {Transm }} \\
& \Theta_{\text {Receiv }} \\
& \Theta_{\text {Equipm }}
\end{aligned}
$$

If only a single metal is used on the receiver side (common equipment side) the $\Theta_{\text {Receiv }}$ can be neglected.

\section{Dynamical Behavior}

If any temperature which is measured changes, the corresponding thermo voltage will change too. This leads to a required recalculation of the output voltage. It has to be done as often as a measurement will be done. The settling time of the voltage generation needs to be as short as possible. The time of the transmitters measurement including the analog digital conversion $t_{\text {meas }}$, the transmission time itself including data processing inside the network controllers $t_{\text {trans }}$, the time of the measurement of receivers temperature as well as the external temperature $t_{\text {temp }}$, the calculation time of the voltage to be generated $t_{\text {calc }}$ and the stabilization time of the analog output $t_{\text {stab }}$ have to be taken into account to determine the systems delay time $t_{\text {delay }}(1)$.

$t_{\text {meas }}+t_{\text {trans }}+t_{\text {temp }}+t_{\text {calc }}+t_{\text {stab }}=t_{\text {delay }}$

In areas of good radio link the calculation time $t_{\text {calc }}$ determines the delay time. It is proven that 16 thermocouples which are connected to 4 different transmitters will give 16 different independent output voltages at a measuring interval of $5 \mathrm{~Hz}$. This leads to a single channel delay time of maximum $12.5 \mathrm{~ms}$. In areas with strong and changing radio interference the transmission time $t_{\text {trans }}$ determines the systems delay. Here the systems delay time may rise much higher

\section{Experimental Results}

During calibration process the system based on 4 transmitters with each 4 channels input for thermocouples and one receiver with 16 channels thermo voltage output shows quite ideal behavior. Regarding nearly the complete temperature range of thermocouples type $\mathrm{K}$ from $-100^{\circ} \mathrm{C}$ up to $1100^{\circ} \mathrm{C}$ the deviation is less than $+-0.5 \%$. This is approximately in the same range as the given tolerances of thermocouples (type K) itself.

Figure 3 shows the difference temperature of the receivers analog and digital output. The receiver is set to a temperature of $0^{\circ} \mathrm{C}$. The digital output values are in the range of $\sim+-2^{\circ} \mathrm{C}$ aver the complete range. The analog output values are in the range of $\sim+-4^{\circ} \mathrm{C}$.

At figure 4 the receiver's temperature is set to $65^{\circ}$. Here it's shown that the analog output as well as the digital output is varying in the range of $\sim+-2^{\circ} \mathrm{C}$. 


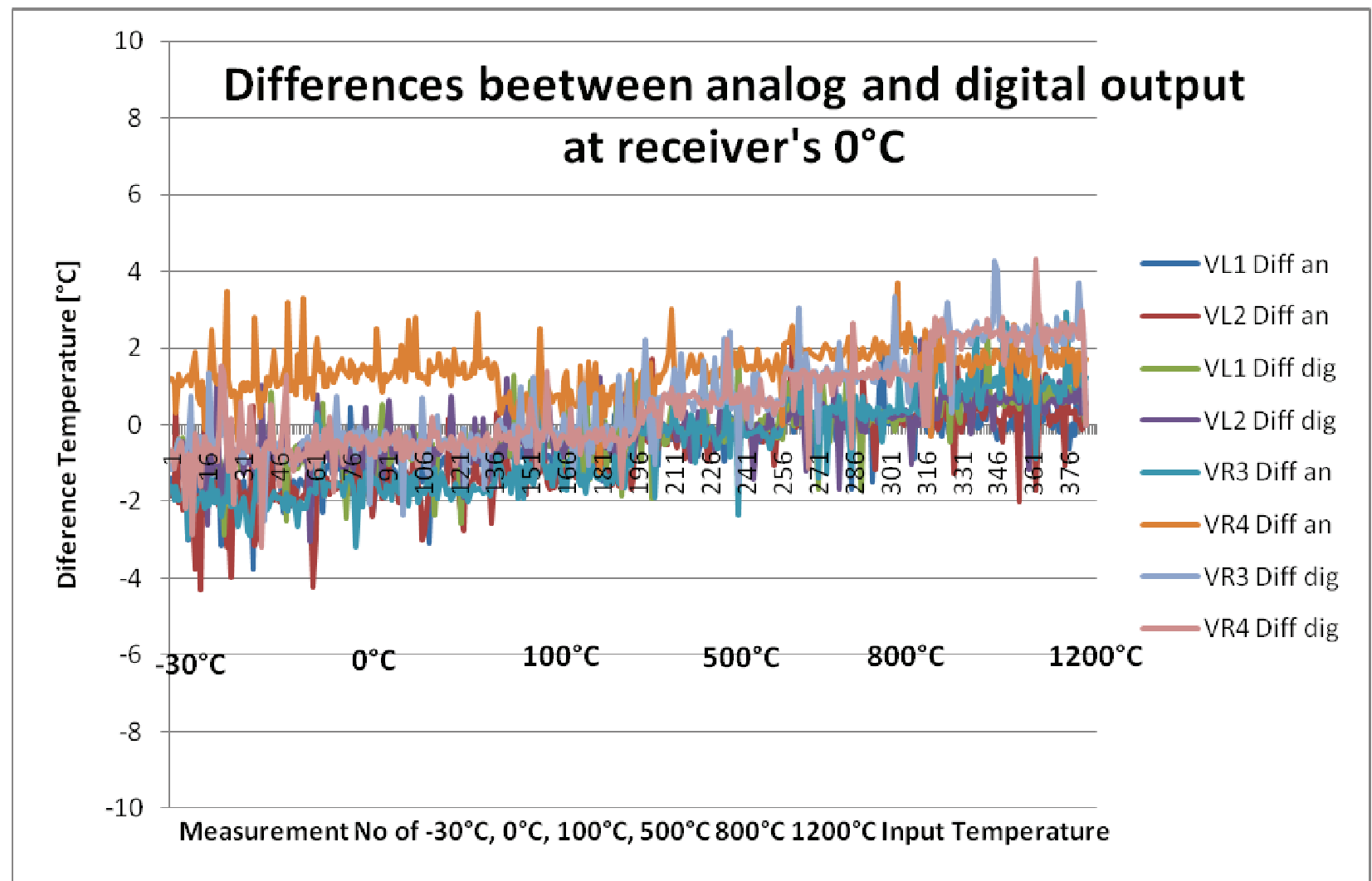

Figure 3: Difference temperatures of digital and analog output values at $0^{\circ} \mathrm{C}$

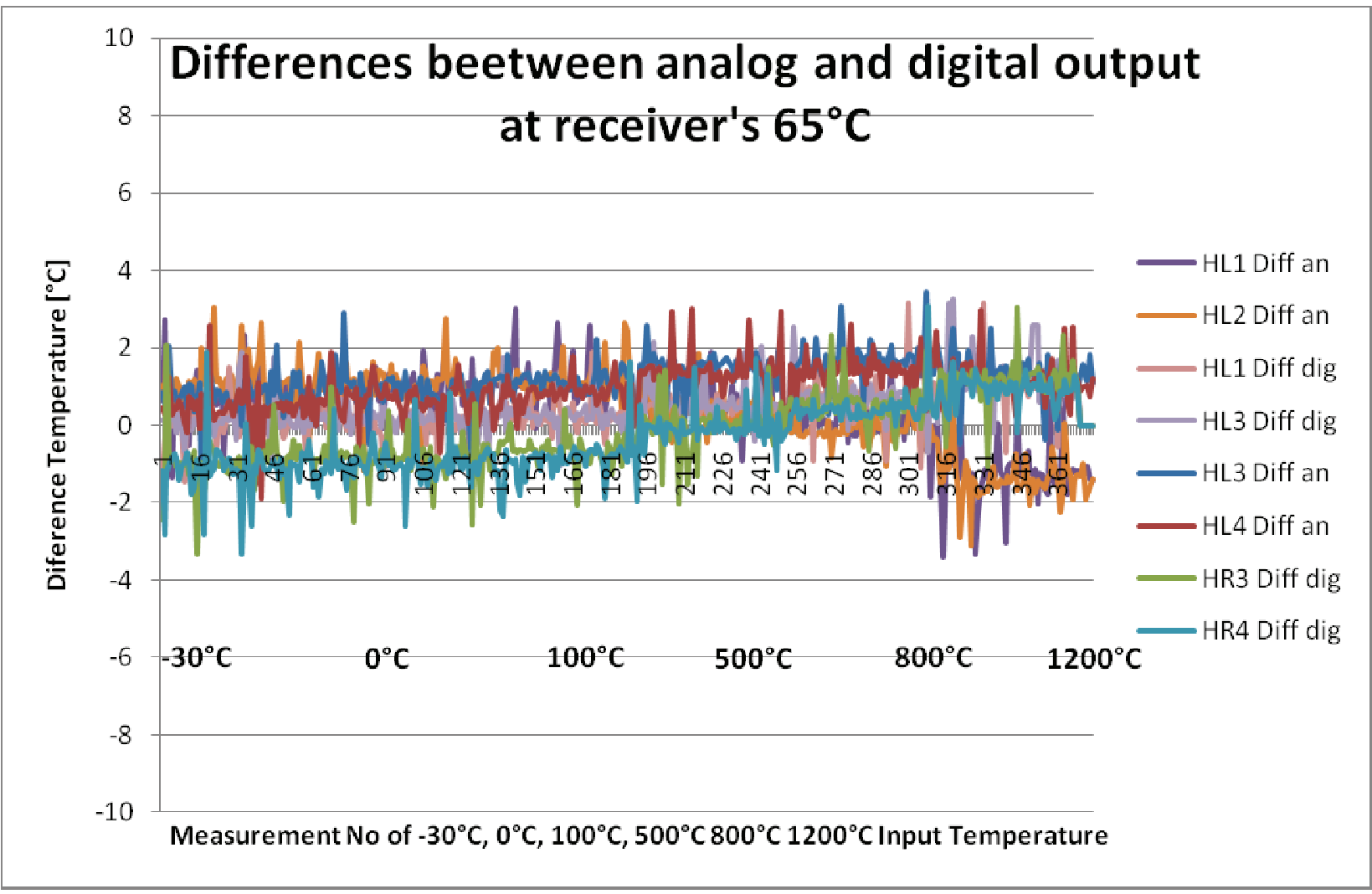

Figure 4: Difference temperature of digital and analog output values at $65^{\circ} \mathrm{C}$ 
In fact the figures 3 and 4 are showing the complete systems behavior including measuring with the transmitters. Here a well calibrated voltage is set as input value for all 16 channels. This voltage varies to fulfill nearly the complete temperature range from $-30^{\circ} \mathrm{C}, 0^{\circ} \mathrm{C}, 100{ }^{\circ} \mathrm{C}, 500^{\circ} \mathrm{C}, 800{ }^{\circ} \mathrm{C}$ up to $1200^{\circ} \mathrm{C}$.

For each temperature value at least 50 measurements were done. Here no averaging was done. If doing averaging the noisy behavior can be eliminated and the performance can be set to much higher accuracy. Of course this will cost time which is possible in applications with slow changing temperatures.

The wireless transmission behavior is proven under extreme conditions of automotive applications. During performance driving tests measurements of the disk brake temperatures were done. Here it was shown that the most disturbing influences are:

fast changing temperatures (very high up to $1000^{\circ} \mathrm{C}$ as well as very low less than $-20^{\circ} \mathrm{C}$ during winter time) fast rotation (up to $2000 \mathrm{rpm}$ )

humidity and strong wetness

salt, gas, oil and dust

fast changing mobile communication systems (UMTS, WLAN, ect.)

Nevertheless the system still works properly even if always external interferences may occur strong delays.

\section{Conclusions}

For a wireless thermocouple - consequently from input to output lots of temperatures as well as their corresponding thermo voltages have to be taken into account. A first system has been presented which offers the user the benefit of modern wireless transmission combined with the usage of common measurement equipment. The wireless system simply replaces the wired connection of a standard thermocouple. It is proven under extreme conditions in automotive applications and is able to connect up to 16 thermocouples.

\section{References}

[1] http://en.wikipedia.org/wiki/Thermocouple

[2] http://www.sentec-elektronik.de 